\title{
Thermosensitive Hydrogel Co-loaded with Gold Nanoparticles and Doxorubicin for Effective Chemoradiotherapy
}

\author{
Tingting Li, ${ }^{1}$ Mingfu Zhang, ${ }^{2}$ Jianzhen Wang, ${ }^{3}$ Tianqi Wang, ${ }^{1}$ Yao Yao, ${ }^{1}$ Xiaomei Zhang, ${ }^{3}$ \\ Cai Zhang, ${ }^{4}$ and Na Zhang ${ }^{1,5}$
}

Received 25 July 2015; accepted 1 September 2015; published online 17 September 2015

\begin{abstract}
Chemoradiotherapy, as a well-established paradigm to treat various cancers, still calls for novel strategies. Recently, gold nanoparticles (AuNPs) have been shown to play an important role as a radiosensitizer in cancer radiotherapy. The aim of this study was to evaluate the combination of polyethylene glycol (PEG) modified AuNPs and doxorubicin (DOX) to improve cancer chemoradiotherapy, in which the AuNPs was the radiosensitizer and the DOX was the model chemotherapeutic. A Pluronic ${ }^{\circledR}$ F127-based thermosensitive hydrogel (Au-DOX-Gel) loading AuNPs and DOX was developed by "cold method" for intratumoral injection. The formulation was optimized at a F127 concentration of $22 \%$ for Au-DOX-Gel. The release profiles compared to a control group were assessed in vitro and in vivo. Au-DOX-Gel showed sustained release of AuNPs and DOX. The cell viability and surviving fraction of mouse melanoma (B16) and Human hepatocellular liver carcinoma (HepG2) cells were significantly inhibited by the combination treatment of DOX and AuNPs under radiation. Tumor sizes of mice were significantly decreased by Au-DOX-Gel compared to controls. Interestingly, 3-(4, 5dimethylthiazol-2-yl)-2, 5-diphenyltetrazolium bromide (MTT) assay and Ki-67 staining results showed that tumor cell growth and proliferation were inhibited by AuNPs combined with DOX under radiation, suggesting that the radiosensitization activity and combination effects might be caused by inhibition of tumor cell growth and proliferation. Furthermore, the results of skin safety tests, histological observation of organs, and the body weight changes indicated in vivo safety of Au-DOX-Gel. In conclusion, the AuDOX-Gel developed in this study could represent a promising strategy for improved cancer chemoradiotherapy.
\end{abstract}

KEY WORDS: chemoradiotherapy; doxorubicin; gold nanoparticles; thermosensitive hydrogel.

\section{INTRODUCTION}

Chemoradiotherapy, the concurrent administration of chemotherapeutics and radiation, is a well-established paradigm to treat various cancers clinically (1). As definitive or adjuvant treatment for cancer, chemoradiotherapy has been shown more effective than sole treatment alone, and new strategies for chemoradiotherapy to enhance the cancer therapeutic effect

Electronic supplementary material The online version of this article (doi:10.1208/s12248-015-9828-3) contains supplementary material, which is available to authorized users.

${ }^{1}$ Department of Pharmaceutics, School of Pharmaceutical Science, Shandong University, Jinan, 250012, China.

${ }^{2}$ Key Lab of Colloid and Interface Chemistry, Ministry of Education, Department of Chemistry and Chemical Engineering, Shandong University, Jinan, 250199, China.

${ }^{3}$ Department of Radiation, Qilu Hospital of Shandong University, Jinan, 250012, China.

${ }^{4}$ Institute of Immunopharmacology \& Immunotherapy, School of Pharmaceutical Sciences, Shandong University, Jinan, 250012, China.

${ }^{5}$ To whom correspondence should be addressed. (e-mail: zhangnancy9@sdu.edu.cn) have been reported over the past few years $(2,3)$. Recent advances in radiosensitizers have offered new opportunities for improving the effectiveness of chemoradiotherapy, in that the presence of radiosensitizers in the tumor would enhance the radiation effect (4). For example, a novel delivery system was developed using lipid-polymer nanoparticle formulations of histone deacetylase inhibitors (HDACIs) as radiosensitizers for chemoradiotherapy, resulting in their significantly higher therapeutic efficacy than small-molecule HDACIs in murine models of colorectal and prostate cancer (5).

Numerous radiosensitizers have long been investigated (3,5-7), among which a significant effort is geared towards gold nanoparticles (AuNPs). As a high $Z$ number $(Z=79)$ element, AuNPs have been known to highly absorb X-rays and concentrate radiation absorption. The application of AuNPs as a radiosensitizer has been supported by extensive experimental evidence $(8,9)$. Radio dose-enhancement of $13 \mathrm{~nm}$ AuNPs was investigated on B16F10 melanoma tumor-bearing mice, in which the tumor growth was retarded and the mice survival was prolonged (10). It was also demonstrated that AuNPs could effectively enhance radiation effects on glioblastoma multiforme tumor cells, leading to enhanced DNA damage, delayed tumor growth, and improved survival (6). 
As new strategies for clinical application, combinations of AuNPs and other theranostic agents have drawn substantial attention. A recent study combined gadolinium and AuNPs for radiosensitization and magnetic resonance imaging (MRI)-based resolution, and its results showed improved radiosensitizing effect and reduced cytotoxicity (7). Another application strategy was studied by combination of AuNPs and Arg-Gly-Asp (RGD) peptides, of which the RGD was designed to antagonize the function of $\alpha(v) \beta(3)$ integrin and the AuNPs was used as a radiosensitizer (11). Less tumor progression, less tumor metabolic activity, fewer intratumoral vessels, and less VEGF gene expression were observed as a consequence of molecular targeting therapy and radiotherapy combination. Though several promising combination strategies have been investigated, the study of AuNPs combined with chemotherapeutics for chemoradiotherapy was limited. A recent report presented the hollow gold nanoparticles loading doxorubicin into the hollow cavity for triple combination therapy: chemotherapy, photothermal therapy, and radiotherapy (12). The doxorubicin (DOX) loaded hollow gold nanoparticles enhanced tumor growth delay by a factor of 4.3. These results implied that the combination of AuNPs (with spherical and concrete core) and chemotherapeutics might have great potential for chemoradiotherapy.

As a broad-spectrum anti-cancer chemotherapeutics, DOX was chosen as the model chemotherapeutic in this study. DOX is an anthracycline topoisomerase inhibitor and it could intercalate between base pairs in the DNA helix, resulting in damage to DNA replication, RNA transcription, and protein synthesis. However, the side effects such as cardiotoxicity, hematopoietic suppression, nausea, vomiting, and alopecia have limited its clinical application (13). Thus, a system co-delivering AuNPs and DOX was developed in this study.

For cancer therapy, different routes of administration have been applied clinically, of which the intratumoral injection has been extensively exploited $(14,15)$. Among these numerous studies, the hydrogel has been widely applied owing to its sustained release potential $(16,17)$. In this study, an injectable in situ hydrogel for intratumor delivery was developed and a sustained release profile of the hydrogel was expected. Pluronic ${ }^{\circledR}$ F127 (F127) which has been widely studied as thermosensitive block copolymer was used in the formulations. Approved by the FDA, F127 has been considered to be non-toxic and commercially available. F127 has long been used to form in situ hydrogel to deliver various drugs, such as insulin, urease, interleukin-2, and epidermal growth factor (EGF) (17-19). By forming a depot of AuNPs and DOX, the F127-based thermosensitive hydrogel designed in this study was expected to get an enhanced chemoradiotherapy effect.

In this present study, a novel chemoradiotherapy strategy was reported. A F127 thermosensitive hydrogel (Au-DOXGel) was developed for sustained release of AuNPs and DOX after intratumoral injection. The tumor was then irradiated by X-ray. We hypothesized that an enhanced cancer chemoradiotherapy could be achieved by the AuDOX-Gel: DOX would act as chemotherapeutics while AuNPs would kill tumor cells by enhancing the radiation dose (Fig. 1). The retention time of AuNPs and DOX was investigated, and sustained release profiles were found.

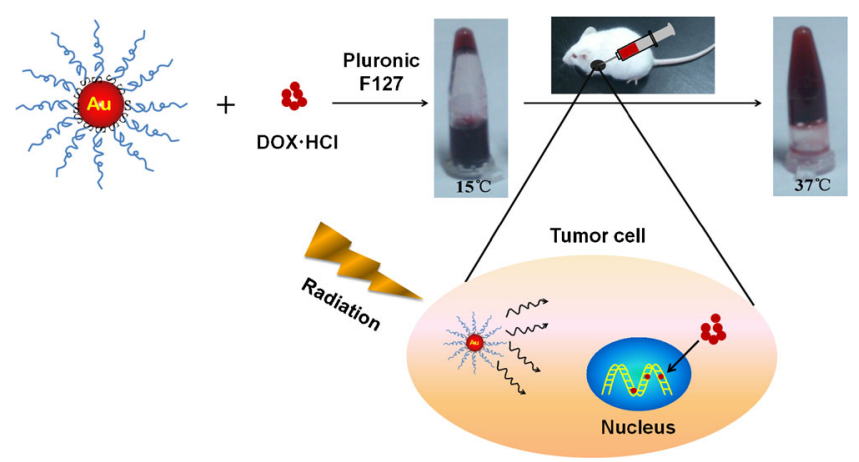

Fig. 1. Pluronic F127 hydrogel co-loaded with gold nanoparticles (AuNPs) and doxorubicin (DOX) for effective cancer chemoradiotherapy. The AuNPs and DOX could be co-loaded into hydrogels and then injected into tumor. After radiation, AuNPs would act as a radiosensitizer and DOX would treat tumor as chemotherapeutics

Substantial radiosensitization effects and combination effects were observed in vitro and in vivo.

\section{MATERIALS AND METHODS}

\section{Reagents}

Tetrachloroauric acid (HAuCl4), Pluronic ${ }^{\circledR}$ F127 (F127), and 3-(4, 5-dimethylthiazol-2-yl)-2, 5-diphenyltetrazolium bromide (MTT) were purchased from Sigma-Aldrich (USA). mPEG-SH (MW 2060 Da, abbreviated as PEG hereafter) were obtained from Shanghai Yare Biotech, Inc. (Shanghai, China). Doxorubicin $\mathrm{HCl}(\mathrm{DOX} \mathrm{HCl}$ ) was purchased from Dalian Meilun Biology Technology Co. Ltd. (Dalian, China). All other reagents were of analytical grade and used without further purification.

\section{Cell Lines and Animals}

Human hepatocellular liver carcinoma (HepG2) and mouse melanoma (B16) cell lines were provided by Institute of Immunopharmacology and Immunotherapy of Shandong University. Male Kunming mice $(20 \pm 2 \mathrm{~g})$ and Wistar rats $(200 \pm 30 \mathrm{~g})$ were provided by Experimental Animal Center of Shandong University. Mice were implanted with B16 cells subcutaneously in their right legs with $1 \times 10^{6}$ cells. The mice received no treatment until the tumor size reached approximately $300 \mathrm{~mm}^{3}$. The radiation was manipulated with high-energy 6-MV photons, by a medical linear accelerator.

The animal experiments in this study were carried out according to the requirements of the National Act on the use of experimental animals (P.R. China) and were approved by Animal Ethics Committee of Shandong University.

\section{Preparation and Characterization of Au-DOX-Gel}

Naked gold nanoparticles were synthesized according to literature (20). Then, the particles were modified by polyethylene glycol (PEG): $1 \mathrm{~mL}$ of $4.12 \%$ PEG was added and the mixture was stirred for $12 \mathrm{~h}$ at room temperature to obtain the PEG-capped AuNPs. The Au-DOX-Gel was prepared according to the "cold method" (18). An appropriate amount 
of F127 granules was added to a solution of AuNPs (1 mg/ $\mathrm{mL})$ containing DOX $(0.5 \mathrm{mg} / \mathrm{mL})$. The mixture was then stirred in an ice bath until the F127 granules were completely dissolved.

Gel formation temperature (GFT) of the hydrogel was determined by tube inversion method. Briefly, each formulation was sealed in a small tube and heated slowly from $10^{\circ} \mathrm{C}$ to $50^{\circ} \mathrm{C}$ at a rate of $1^{\circ} \mathrm{C} / \mathrm{min}$. The sample was equilibrated for $5 \mathrm{~min}$ at each temperature point, and the flowability of each sample was observed by tilting the tubes. The temperature at which the liquid was observed to be immobile was recorded as the GFT. The gelation time (GT) of the samples was determined by transferring the samples from 5 to a $37^{\circ} \mathrm{C}$ water bath. Time measurements of GT were initiated when the sample was placed into a $37^{\circ} \mathrm{C}$ water bath and stopped at the right time when the sample was immobile. The injectability of each formulation was investigated by a 1-mL syringe at different temperatures. The morphology of AuNPs was determined by transmission electron microscopy (TEM) as previously described (21).

\section{Cellular Uptake and MTT Assay}

Cells were seeded on six-well plates. AuNPs were added to the culture medium at a terminal concentration of $5 \mathrm{nM}$. Cells were harvested at certain intervals after which the $\mathrm{Au}$ mass was determined by inductively coupled plasma atomic emission spectroscopy (ICP-AES). MTT assay was employed for cell viability studies and was carried out as previously described (21).

\section{Colony Formation Assay}

Cells were seeded in six-well plates using different numbers of cells, in accordance with gradient doses of radiation. The cells were then incubated with AuNPs (5 nM) with or without DOX before irradiation. After 10 days, cell colonies were counted. Plating efficiency (PE) was calculated relative to the control group (0 Gy), and the survival fraction (SF) of each group was determined using the equation: $\mathrm{SF}=$ colony number $/($ plating cell number $\times \mathrm{PE})$. The data were fitted to the single-hit multi-target statistical model where the SF at different radiation doses (D) is given as $\mathrm{SF}=$ $1-(1-\exp (-k \times D))^{N}$, where $k=1 / D_{0} . \quad D_{0}$ describes the straight portion of the surviving curve and is the dose required to kill $63 \%$ of cells. $N$ is the $y$ intercept of the surviving curve extrapolated back to the $y$-axis. Using the SF equation, other parameters including SF2 (surviving fraction of 2 Gy) and Dq (quasi-threshold dose) could be calculated (22). The sensitizing enhance rate (SER) of Dq and SF2 was calculated as below: $\mathrm{SER}_{\mathrm{Dq}}=\mathrm{Dq}$ of "IR" group/Dq of "IR + $\mathrm{Au}$ " or "IR + Au+DOX" group; $\mathrm{SER}_{\mathrm{SF} 2}=\mathrm{SF} 2$ of "IR" group/ $\mathrm{SF} 2$ of "IR $+\mathrm{Au}$ " or "IR $+\mathrm{Au}+\mathrm{DOX}$ " group, where IR, Au, and DOX denote irradiated, AuNPs treated, and DOX treated, respectively.

\section{In Vitro Release Profiles of DOX from Au-DOX-Gel}

The in vitro release profiles of DOX from different formulations were investigated by dialysis method with PBS of $\mathrm{pH} 6.8$ or $\mathrm{pH} 7.4$ at $37^{\circ} \mathrm{C}$.

\section{Determination of AuNPs and DOX in Tumor}

AuNPs in collected tumors were determined as follows: tumors were weighted, mixed with ethanol, and homogenized by high-speed shearing machine. Then, the homogenates were heated at $110^{\circ} \mathrm{C}$ in an oil bath to evaporate the ethanol. The precipitates were then completely lysed in $2 \mathrm{~mL}$ freshly made aqua regia for $5 \mathrm{~min}$, diluted with $6 \mathrm{~mL}$ water before analysis by ICP-AES (23). DOX in collected tumors were determined as previously described (24). The distribution of DOX in tumor was further evaluated by ex vivo imaging. Tumors were washed three times by PBS before being subjected to ex vivo fluorescence imaging (IVIS Kinetic, Caliper, USA).

\section{Anti-tumor Activity}

Mice in each group were injected intratumorally with $0.1 \mathrm{~mL}$ of each formulation containing AuNPs (0.1 mg per tumor) or DOX (0.05 mg per tumor) or both, and saline was used as control. The tumors were then radiated ( 2 Gy per tumor) $30 \mathrm{~min}$ after injection to make sure the hydrogel was well formed. The mice in each group received injections weekly and radiation three times a week. After first injection, the mice were observed for an additional 20 days. The tumor size and body weight of each mouse was monitored every 2 days and the mice were sacrificed on the 20 th day.

\section{Immunohistochemistry and Histological Evaluation}

At the 20th day after first injection, tumors, heart, liver, spleen, lung, and kidneys were excised. The Ki-67 staining of tumor tissues was carried out according to the literature (25). The heart, liver, spleen, lung, and kidneys were stored in $10 \%$ formalin and embedded in paraffin. Then, $3-\mu$ m-thick sections were made and stained with hematoxylin-eosin (H\&E).

\section{Skin Safety}

Wistar rats $(200 \pm 30 \mathrm{~g})$ were employed in skin safety test. The backside of rat was clearly shaved before subcutaneous injection of $0.5 \mathrm{~mL}$ AuNPs loaded hydrogel ( $0.5 \mathrm{mg} \mathrm{Au})$. The skin was then photographed at a predetermined time.

\section{Statistical Analysis}

All studies were repeated three times and measured in triplicate at least. Either Student's $t$ test or a one-way analysis of variance (ANOVA) was used to evaluate the data with $p<0.05$ as the minimal level of significance.

\section{RESULTS}

\section{Characterization of Au-DOX-Gel}

The UV-vis spectra of naked AuNPs and PEG-layered AuNPs showed an absorbance maximum at 518 and $524 \mathrm{~nm}$, respectively, by surface plasmon resonance (SPR) (Fig. 2a) (26). The morphology of AuNPs manifested a homogenous shape with a mean diameter of $13.09 \pm 1.77 \mathrm{~nm}$ (Fig. 2b). As shown in Fig. 1, the hydrogel was flowable at a low 
a

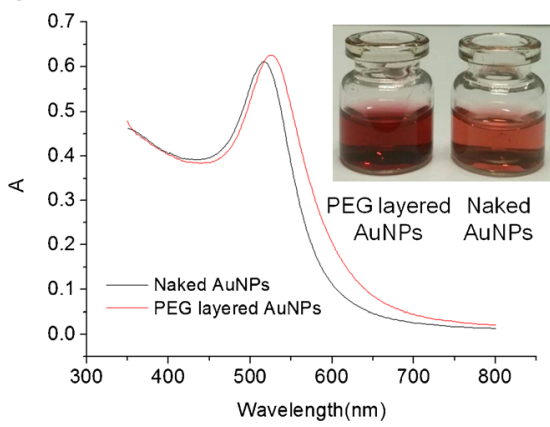

b

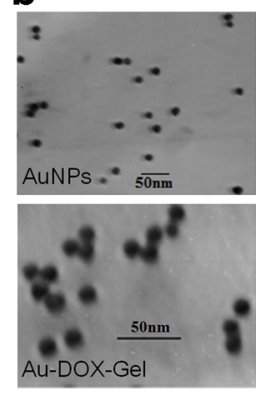

Fig. 2. Characterization of AuNPs and Au-DOX-Gel. a UV-vis spectra of naked AuNPs and PEG-layered AuNPs. b Morphology of AuNPs (PEG layered) and Au-DOX-Gel measured by transmission electron microscopy (TEM)

temperature $\left(15^{\circ} \mathrm{C}\right)$ and turned into a jelly-like appearance at body temperature $\left(37^{\circ} \mathrm{C}\right)$. Characterization results of formulations with different F127 concentrations were presented in Table I, showing that the optimal formulation of Au-DOXGel contains F127 at a concentration of $22 \%$ (20\% for blank hydrogel).

\section{Radiosensitization Effects of AuNPs on Cell Lines}

The uptake of AuNPs by B16 and HepG2 cells was investigated first, as shown in Fig. 3. For B16, the maximal internalization rate of AuNPs was $48.5 \%$ at $24 \mathrm{~h}$ and HepG2 was $41.9 \%$ at $36 \mathrm{~h}$. The difference of cellular uptake rates by HepG2 between 24 and $36 \mathrm{~h}$ was not statistically significant $(p>0.05)$. Therefore, the radiation time was set at $24 \mathrm{~h}$ after AuNPs were added for the following MTT assay and colony formation assay.

The concentrations of AuNPs used in this study had no obvious effect on cell viability towards B16 and HepG2, as seen in Fig. 4b. Statistical results in Fig. 4a showed that the 5-

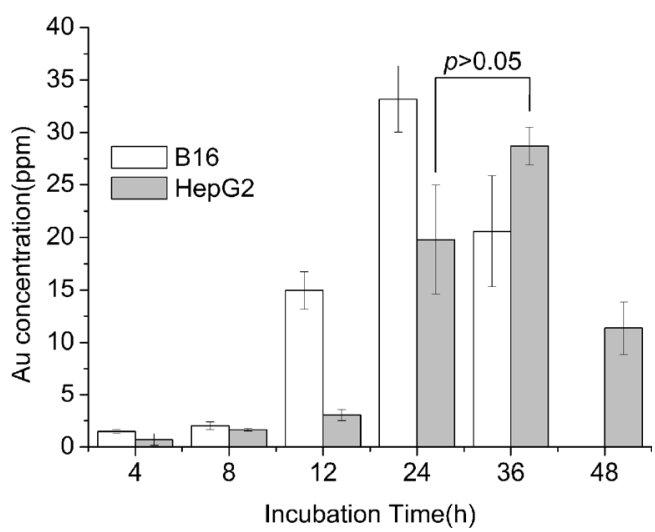

Fig. 3. Cellular uptake of AuNPs in B16 and HepG2. AuNP concentration was $5 \mathrm{nM}(\sim 68.42 \mathrm{ppm}$ of $\mathrm{Au})$

$\mathrm{nM}$ AuNPs combined with radiation significantly inhibited cell growth compared to radiation alone at given doses of $\mathrm{X}$ ray on both cell lines. From our preliminary work, it was found that the inhibition of cell growth was related to concentrations of AuNPs as well as X-ray doses. Sensitization rates at different treatments were carried out in order to examine the relationship between AuNP concentration, radiation dose, and the sensitization effect (Table II). It was noteworthy that the sensitization rates of AuNPs have a positive correlation with concentrations of AuNPs but not much correlation with X-ray doses.

The surviving fractions were determined at each radiation dose, measured by colony formation assay. As shown in Fig. 4e, f, for both cells, the X-ray induced decreased surviving fraction as the radiation dose increased. Surviving fractions decreased significantly when both cells were incubated with AuNPs before radiation $(p<0.05)$. As shown in Table III, The Dq and SF2 for both cell lines were decreased after AuNPs were combined and the SER

Table I. Characterization of Blank Gel, Au-Gel, and Au-DOX-Gel with Various F127 Concentrations

\begin{tabular}{|c|c|c|c|c|c|c|c|c|c|c|c|c|c|}
\hline & & \multicolumn{12}{|c|}{ Concentration of F127 gel $(w / w)$} \\
\hline & & \multicolumn{3}{|c|}{$16 \%$} & \multicolumn{3}{|c|}{$20 \%$} & \multicolumn{3}{|c|}{$22 \%$} & \multicolumn{3}{|c|}{$24 \%$} \\
\hline & & Blank & $\mathrm{Au}$ & $\begin{array}{c}\mathrm{Au}- \\
\mathrm{DOX}\end{array}$ & Blank & $\mathrm{Au}$ & $\begin{array}{c}\mathrm{Au}- \\
\mathrm{DOX}\end{array}$ & Blank & $\mathrm{Au}$ & $\begin{array}{l}\mathrm{Au}- \\
\mathrm{DOX}\end{array}$ & blank & $\mathrm{Au}$ & $\begin{array}{c}\mathrm{Au}- \\
\mathrm{DOX}\end{array}$ \\
\hline \multirow{2}{*}{\multicolumn{2}{|c|}{$\operatorname{GFT}\left({ }^{\circ} \mathrm{C}\right)$}} & 35.5 & $>50$ & $>50$ & 23.8 & 37.3 & 37.2 & 20.3 & 27.8 & 27.2 & 18.3 & 23.3 & 22.2 \\
\hline & & \pm 0.9 & -50 & -50 & \pm 0.3 & \pm 0.3 & \pm 0.3 & \pm 0.6 & \pm 0.3 & \pm 0.3 & \pm 0.6 & \pm 0.3 & \pm 0.3 \\
\hline \multicolumn{2}{|c|}{ GT(s) } & $\begin{array}{c}127 \pm \\
13\end{array}$ & - & - & $50 \pm 2$ & $\begin{array}{c}263 \pm \\
13\end{array}$ & $\begin{array}{c}242 \pm \\
12\end{array}$ & $39 \pm 5$ & $\begin{array}{c}166 \pm \\
10\end{array}$ & $\begin{array}{c}170 \pm \\
9\end{array}$ & $18 \pm 1$ & $\begin{array}{c}113 \pm \\
7\end{array}$ & $\begin{array}{c}120 \pm \\
2\end{array}$ \\
\hline \multirow{3}{*}{$\begin{array}{l}\text { Injecta } \\
\text {-bility }\end{array}$} & $10^{\circ} \mathrm{C}$ & + & + & + & + & + & + & + & + & + & \pm & + & + \\
\hline & $20^{\circ} \mathrm{C}$ & + & + & + & + & + & + & \pm & + & + & - & \pm & \pm \\
\hline & $25^{\circ} \mathrm{C}$ & + & + & + & \pm & + & + & \pm & \pm & \pm & - & - & - \\
\hline
\end{tabular}

Red box reflected the optimal formulation of Au-DOX-Gel and blank hydrogel, respectively

GFT gel formation temperature, GT gelation time, $A u$ AuNPs loaded hydrogel, $A u-D O X$ AuNPs and DOX co-loaded hydrogel

"+": injectable (the sample could get through the syringe needle smoothly)

" \pm ": less injectable (the sample could get through the syringe needle with some resistance)

"-": uninjectable (the sample could not get through the syringe needle) 
a

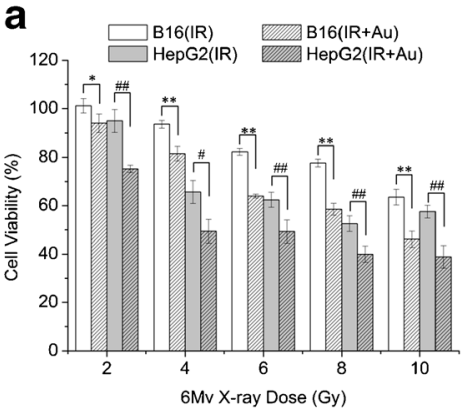

C

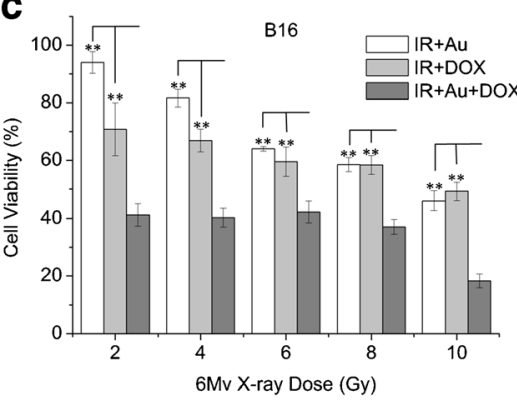

e

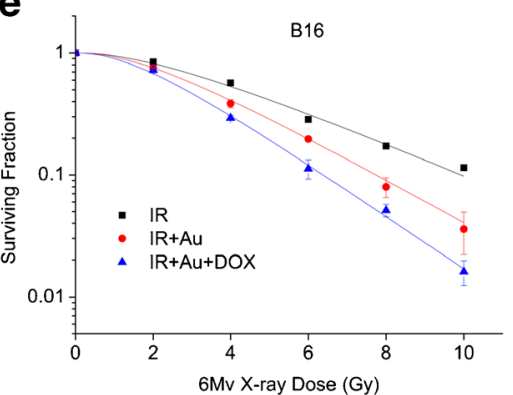

b

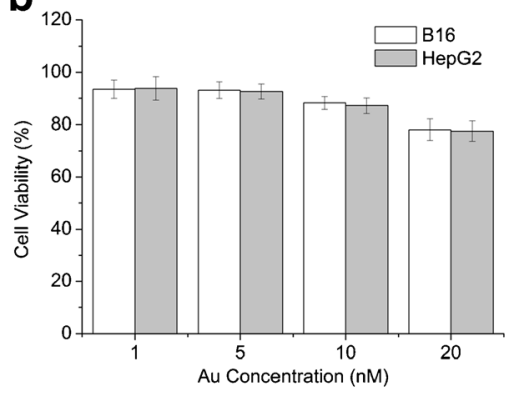

d

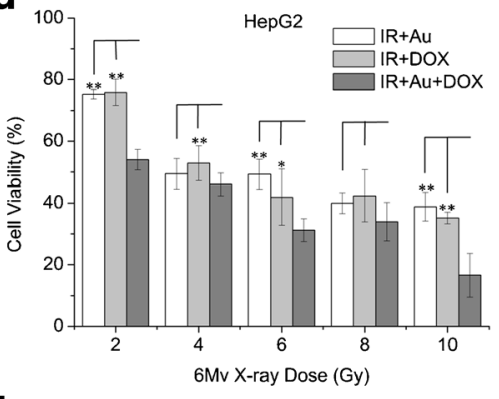

f

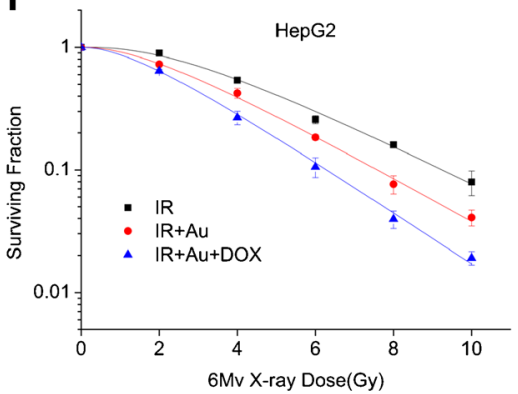

Fig. 4. a Sensitization ability of AuNPs $(5 \mathrm{nM})$ under various doses of X-ray on B16 and HepG2 cell lines, measured by MTT assay. $* p<0.05$, ${ }^{* *} p<0.01$, statistically significance between IR and IR+Au treatment on B16 cells; ${ }^{\#} p<0.05,{ }^{\# \#} p<0.01$, statistically significance between IR and IR + Au treatment on HepG2 cells. b Cytotoxicity of AuNPs at different concentrations on B16 and HepG2 cell lines, measured by MTT assay. Combination effect of AuNPs $(5 \mathrm{nM})$ and DOX (20 nM for B16 and $200 \mathrm{nM}$ for HepG2) under various doses of X-ray on B16 cells (c) and HepG2 cells (d), measured by MTT assay. ${ }^{*} p<0.05,{ }^{* *} p<0.01$, statistically significance between sole (Au or DOX) treatment and combination treatment under radiation. Surviving fraction of B16 cells (e) and HepG2 cells (f) incubated with AuNPs (5 nM) or DOX (20 nM for B16 and $200 \mathrm{nM}$ for HepG2) or both, under various doses of X-ray, measured by colony formation assay. "IR" was for groups with irradiation only. "IR+Au" was for groups incubated with AuNPs before irradiation. "IR+DOX" was for groups incubated with DOX before irradiation. "IR+ $\mathrm{Au}+\mathrm{DOX} "$ was for groups incubated with AuNPs and DOX before irradiation.

for both cell lines were more than 1. The higher the Dq and SF2 were, the more radioresistant the cells. An SER higher than 1 meant the treatment cells were radiosensitive and the higher the SER was, the more radiosensitive the cells.

\section{Combination Effects of AuNPs and DOX Under Radiation on Cell Lines}

The results of cell growth inhibition of combination treatment were shown in Fig. 4c, d, measured by MTT assay.

Table II. Sensitization Rate (\%) of AuNPs at Different Concentrations

\begin{tabular}{|c|c|c|c|c|c|c|c|c|c|}
\hline & & \multicolumn{8}{|c|}{ Final concentration of $\operatorname{AuNPs}(\mathrm{nM})$} \\
\hline & & \multicolumn{2}{|l|}{1} & \multicolumn{2}{|l|}{5} & \multicolumn{2}{|l|}{10} & \multicolumn{2}{|l|}{20} \\
\hline & & HepG2 & B16 & HepG2 & B16 & HepG2 & B16 & HepG2 & B16 \\
\hline \multirow[t]{5}{*}{ 6-MV X-ray dose/Gy } & 2 & 15.80 & 5.96 & 20.69 & 7.11 & 38.64 & 24.90 & 45.77 & 31.98 \\
\hline & 4 & 10.62 & 6.57 & 24.71 & 12.77 & 35.41 & 19.69 & 34.93 & 31.42 \\
\hline & 6 & 18.28 & 10.78 & 21.01 & 22.27 & 41.17 & 36.27 & 43.65 & 44.57 \\
\hline & 8 & 15.90 & 16.04 & 23.92 & 24.69 & 31.42 & 40.18 & 45.71 & 60.31 \\
\hline & 10 & 26.41 & 13.27 & 32.49 & 27.42 & 44.73 & 47.53 & 57.39 & 53.03 \\
\hline
\end{tabular}

The sensitization rate of AuNPs was calculated as sensitization rate (\%)=(viability of "IR"-viability of "IR+Au")/viability of "IR" $\times 100 \%$ $A u N P s$ gold nanoparticles, HepG2 human hepatocellular liver carcinoma cell lines, B16 mouse melanoma cell lines 
Table III. Related Biological Parameters of Cell Surviving Curve Under Different Treatments

\begin{tabular}{|c|c|c|c|c|c|c|}
\hline & \multicolumn{2}{|l|}{ IR } & \multicolumn{2}{|l|}{$\mathrm{IR}+\mathrm{Au}$} & \multicolumn{2}{|c|}{$\mathrm{IR}+\mathrm{Au}+\mathrm{DOX}$} \\
\hline & HepG2 & B16 & HepG2 & B16 & HepG2 & B16 \\
\hline$k$ & 0.362 & 0.309 & 0.408 & 0.407 & 0.481 & 0.498 \\
\hline$N$ & 2.941 & 2.214 & 2.278 & 2.404 & 2.118 & 2.469 \\
\hline $\mathrm{Dq}$ & 2.980 & 2.572 & 2.018 & 2.155 & 1.560 & 1.815 \\
\hline SF2 & 0.897 & 0.845 & 0.729 & 0.755 & 0.644 & 0.729 \\
\hline $\mathrm{SER}_{\mathrm{Dq}}$ & - & - & 1.477 & 1.194 & 1.910 & 1.417 \\
\hline $\mathrm{SER}_{\mathrm{SF} 2}$ & - & - & 1.230 & 1.119 & 1.393 & 1.160 \\
\hline
\end{tabular}

$I R$ radiation, $A u$ gold, $D O X$ doxorubicin, $H e p G 2$ human hepatocellular liver carcinoma cell lines, $B 16$ mouse melanoma cell lines, $D q$ quasithreshold dose, $S F 2$ surviving fraction of $2 \mathrm{~Gy}, S E R$ sensitizing enhance rate

For B16 cell lines used in this study, the combination treatment showed much lower cell viabilities compared to single treatment $(p<0.01)$. For HepG2, the combination effect was less evident; statistical significance was only observed in 2, 6, and 10 Gy radiation dose. The surviving fractions at each radiation dose, measured by colony formation assay, were shown in Fig. 4e, f. Radiation combined with AuNPs in the presence of DOX induced greater decrease in surviving rates compared to sole treatment $(p<0.05)$. As shown in Table III, the Dq and SF2 for both cell lines were decreased more compared to sole treatment. Correspondingly, the SER for both cell lines were higher in combination treatment than in sole treatment.

\section{In Vitro Release Profiles of DOX and Tumor Retention of AuNPs and DOX}

The release profiles of DOX were illustrated in Fig. 5a, b. The release of DOX from aqueous solutions reached the maximal point at about $8 \mathrm{~h}$, and the release accumulation at $8 \mathrm{~h}$ was statistically more than that in corresponding hydrogel formulation groups $(p<0.05)$. In contrast, it took about $12 \mathrm{~h}$ for hydrogel formulations to get the maximal point. No significant difference was found at every single time point between the groups of "DOX-Gel" and "Au-DOX-Gel" ( $p>0.05)$, nor between the groups of "DOX- $\mathrm{H}_{2} \mathrm{O}$ " and "Au-DOX- $\mathrm{H}_{2} \mathrm{O}$ " $(p>0.05)$. The release profiles in $\mathrm{pH} 7.4$ and $\mathrm{pH} 6.8$ were not significantly different $(p>0.05)$.

In order to find out whether sustained release also occurred in vivo, the tumor retention of AuNPs and DOX was evaluated. As shown in Fig. 5c, d, DOX was released from solution soon after injection and only $33.7 \%$ remained in the tumor at $8 \mathrm{~h}$. In contrast, DOX in hydrogel group released much more slowly, remaining $58.7 \%$ in tumor at $24 \mathrm{~h}$ and $15.7 \%$ at $48 \mathrm{~h}$. AuNPs released relatively slowly than DOX from each formulation. The retention rate of AuNPs was 35.8 and $14.2 \%$ at $48 \mathrm{~h}$ for hydrogel and solution, respectively.

\section{In Vivo Anti-tumor Activity}

The radiosensitization activity of AuNPs could be seen from Fig. 6a, b. The tumor sizes of the IR $+A u$ group were significantly decreased compared to the saline group $(p<0.01)$ and irradiation group $(p<0.01)$. The tumor sizes were decreased more in the combination treatment than in the sole treatment $(p<0.01)$. From Fig. $6 \mathrm{a}, \mathrm{b}$, it could be clearly seen that the tumor volume inhibition in the co-loaded hydrogel group was significantly increased compared to solution mixture group. The X-ray dose used in the tests slightly decreased the tumor sizes, and the differences showed no statistical significance compared to the saline group ( $p=$ 0.07). The AuNPs loaded hydrogel without radiation showed no significant difference on tumor volume compared to the saline group $(p=0.07)$ Ki-67 staining was then performed to find the changes in degree of proliferation in tumor tissue (Fig. 6d). Tumor in the saline group was stained as control, suggesting a high proliferation status. The proliferation was inhibited after the tumors were treated by radiation combined with AuNPs, as the stains were less than that in the control group. More inhibition of proliferation was observed in the IR+ $\mathrm{Au}+\mathrm{DOX}(\mathrm{G})$ group. IR, Au, and DOX denote irradiated, AuNPs treated, and DOX treated, respectively.

\section{Primary Evaluation of Safety}

Body weight could be one of the primary indicators of safety (27). It could be seen from Fig. $6 c$ that the concentrations of AuNPs and DOX applied in this study did not affect the body weight compared to the saline group $(p>0.05)$. However, solution groups with DOX at $0.05 \mathrm{mg}$ per mice decreased the body weights of mice significantly compared to the control group $(p<0.05)$. Skin safety was testified, showing no obvious injury or irritation on the skin surface (Fig. S). Histological analysis of heart, liver, spleen, lung, and kidneys showed that no visible tissue damage, inflammation, or lesions were observed (Fig. 7a, b) (28).

\section{DISCUSSION}

Cancer remains a major health burden throughout the world, and novel strategies in chemoradiotherapy are still needed (29-31). F127-based Au-DOX-Gel formulated and demonstrated its superior properties for intratumoral delivery. F127 was the key material for hydrogel formation and its gelation manner relied on its concentrations (32). Results showed that the preparations needed slightly more F127 (2 g per $100 \mathrm{~mL}$ ) to form hydrogel at a certain condition when loaded with AuNPs. Previous literature reported that the hydrophilic homopolymer with medium molecular weight "melts" the F127 hydrogel (33). The PEG used in this study was medium molecular (2060 Da) and the PEG was a hydrophilic homopolymer. Therefore, the changes of melting behavior of the hydrogel after being loaded with AuNPs were 

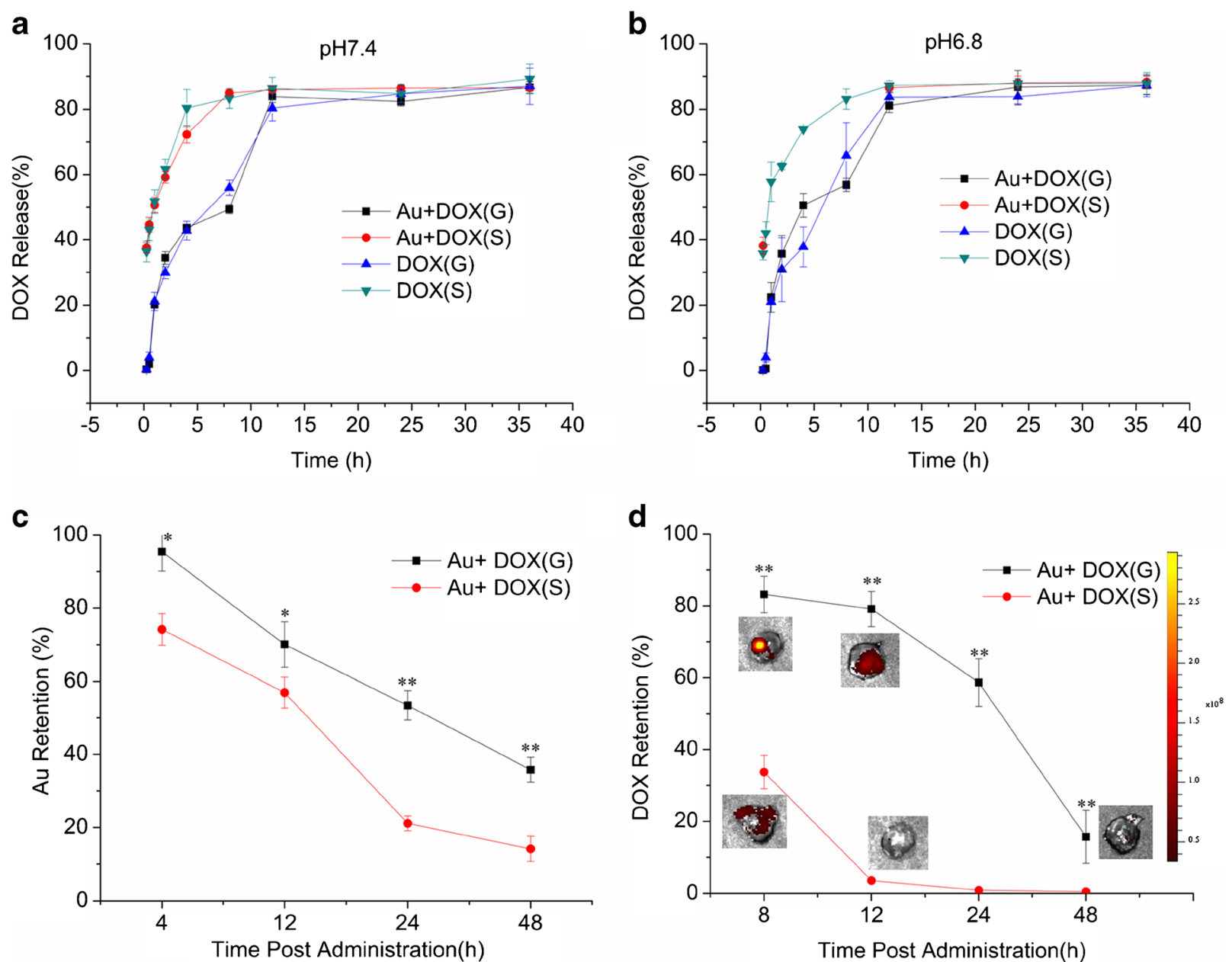

Fig. 5. Release profiles of DOX from different formulations in PBS at pH 7.4 (a) and pH 6.8 (b). PBS of pH 7.4 was to mimic the body environment and $\mathrm{PBS}$ of $\mathrm{pH} 6.8$ was to mimic the slightly acidic environment in tumor. All tests were carried out at $37^{\circ} \mathrm{C}$. $\mathbf{c}$ AuNP retention in tumor at different times after administration, measured by ICP-AES. d DOX retention in tumor at different times after administration, measured by fluorescence imaging system and spectrofluorophotometer, respectively. "Au+DOX $(\mathrm{G})$ " was for Au-DOX-Gel. "Au+DOX(S)" was for AuNPs solution and DOX solution mixture. "DOX $(\mathrm{G})$ " was for DOX loaded hydrogel. "DOX $(\mathrm{S})$ " was for DOX solution. * $p<0.05$, ${ }^{* *} p<0.01$, statistically significance between $\mathrm{Au}+\operatorname{DOX}(\mathrm{G})$ and $\mathrm{Au}+\mathrm{DOX}(\mathrm{S})$

probably due to the PEG layer on the surface of AuNPs. AuDOX-Gel was expected to form a hydrogel at body temperature and then gradually release "drugs." As can be seen from Fig. 1, the Au-DOX-Gel could be injectable at $15^{\circ} \mathrm{C}$ and could form hydrogel at $37^{\circ} \mathrm{C}$. Considering together with its gelation manner in Table I, we could conclude that the Au-DOX-Gel was injectable at low temperature and could form hydrogel when injected into tumor $\left(\geq 37^{\circ} \mathrm{C}\right)$. Similar gelation manner was also reported by literature (19).

The in vitro release profiles of DOX confirmed the hypothesis that the DOX would release more slowly in hydrogel than in aqueous solution, which was consistent with literature (34). As a clarification, only the release of DOX was tested because AuNPs could not go through the dialysis bag. The release media was carried out at $\mathrm{pH} 7.4$ and $\mathrm{pH} 6.8$ to mimic the physiological $\mathrm{pH}$ and the $\mathrm{pH}$ in tumor, respectively. Similar results were found in Fig. S, from which it could be seen that the hydrogel was half-disappeared at $12 \mathrm{~h}$ and almost totally disappeared at $24 \mathrm{~h}$. The release profiles of DOX and AuNPs were further investigated in vivo. Results showed a more retard release behavior of the hydrogel than solution and the retentions at $48 \mathrm{~h}$ were $15.7 \%$ and $35.8 \%$ for DOX and AuNPs, respectively.
B16 and HepG2 cell lines were tested in vitro to evaluate the radiosensitization activity of AuNPs and the combination treatment strategy. DOX is a broad-spectrum anti-cancer drug and was reported to cure many kinds of cancers, including numerous reports for melanoma and hepatic carcinoma (13,35-37). The cellular uptake results presented that $\mathrm{Au}$ concentration decreased after reaching the peak concentration, which could be explained by the exocytosis of AuNPs in the later stage (38). For biocompatibility, the AuNPs were coated with PEG which is extensively used to improve the stability in biological media (8). Cell viability and tumor volume inhibition results revealed that the AuNPs had no obvious toxicity at the concentrations used in this study which was consistent with other reports (6).

Cell growth inhibition was evaluated by MTT assay. The half maximal inhibitory concentration (IC50) of DOX was measured. The average IC50 of DOX for B16 and HepG2 were 0.21 and $4.2 \mu \mathrm{M}$, respectively. According to the MTT manufacturer's instruction, the accuracy is dependable only when the absorbance is between 0.2 and 0.9 . The absorbance in MTT assay depends on the numbers of living cells (cell viability). Therefore, different DOX concentrations were 


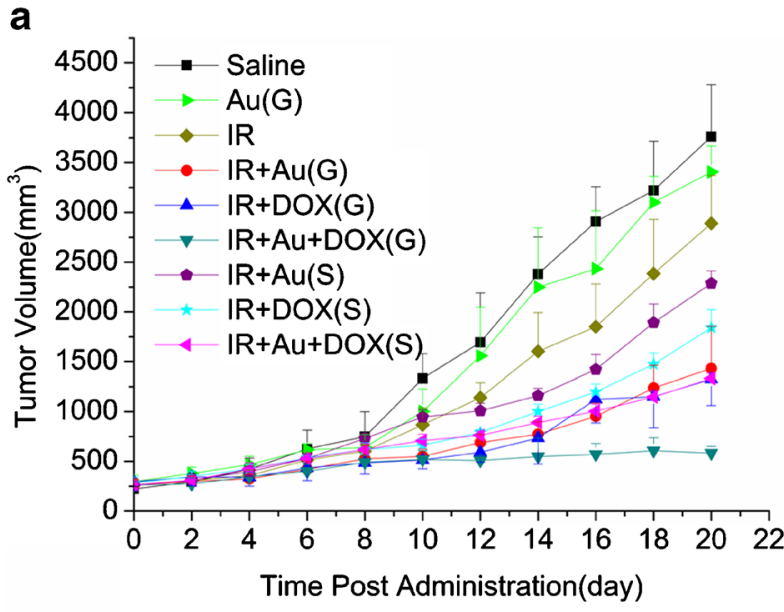

C

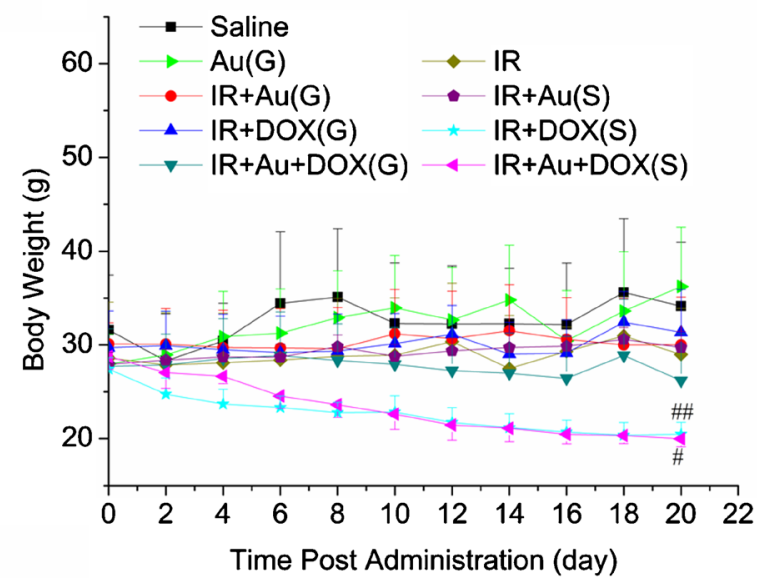

b

Tumor volumes in each group at $20^{\text {th }}$ day post administration.

\begin{tabular}{lcccc}
\hline \multicolumn{1}{c}{ Group } & Volume $\left(\mathrm{mm}^{3}\right)$ & $p^{1}$ & $p^{2}$ & $p^{3}$ \\
\hline Saline & $3761 \pm 519$ & - & $<0.01$ & - \\
$\mathrm{Au}(\mathrm{G})$ & $3408 \pm 261$ & 0.07 & $<0.01$ & - \\
$\mathrm{IR}$ & $2889 \pm 482$ & 0.07 & $<0.01$ & - \\
$\mathrm{IR}+\mathrm{Au}(\mathrm{G})$ & $1429 \pm 427$ & $<0.01$ & $<0.01$ & - \\
$\mathrm{IR}+\mathrm{DOX}(\mathrm{G})$ & $1326 \pm 266$ & $<0.01$ & $<0.01$ & - \\
$\mathrm{IR}+\mathrm{Au}+\mathrm{DOX}(\mathrm{G})$ & $583 \pm 67$ & $<0.01$ & - & - \\
$\mathrm{IR}+\mathrm{Au}(\mathrm{S})$ & $2287 \pm 126$ & 0.02 & $<0.01$ & $<0.01$ \\
$\mathrm{IR}+\mathrm{DOX}(\mathrm{S})$ & $1841 \pm 180$ & $<0.01$ & $<0.01$ & $<0.01$ \\
$\mathrm{IR}+\mathrm{Au}+\mathrm{DOX}(\mathrm{S})$ & $1331 \pm 51$ & $<0.01$ & $<0.01$ & - \\
\hline
\end{tabular}

$p^{1}: p$ value vs. Saline group

$p^{2}: p$ value vs. $\mathrm{IR}+\mathrm{Au}+\mathrm{DOX}(\mathrm{G})$ group $p^{3}: p$ value vs. IR+Au+ DOX(S) group

d

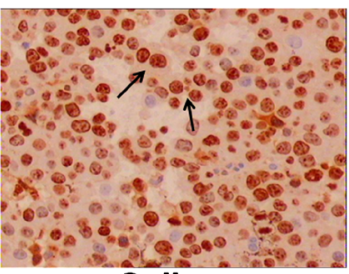

Saline

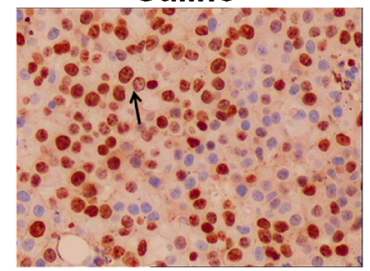

IR+DOX(G)

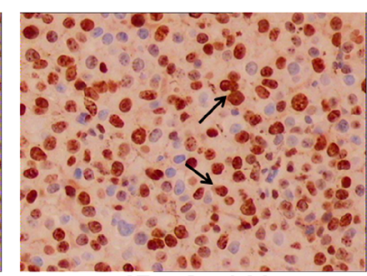

IR+Au(G)

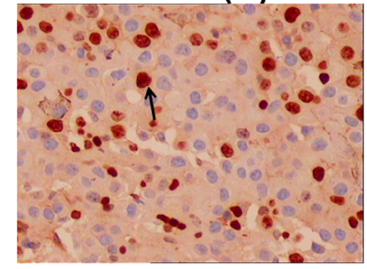

$\mathrm{IR+Au+DOX(G)}$

Fig. 6. a Changes in tumor volumes of B16 tumor-bearing mice after first treatment. b Tumor volumes in each group at 20 th day post administration. Tumor volume was estimated as half of the small diameter squared times the large diameter. $\mathbf{c}$ Changes in body weights of B16 tumor-bearing mice after first treatment. d The Ki-67 immunohistochemical stain of tumor tissue at 20th day after first treatment, representing the changes of cell proliferation in tumor tissue. $A u(G)$ AuNPs loaded hydrogel, $I R$ radiation, $I R+A u(G)$ AuNPs loaded hydrogel with radiation, $I R+D O X(G)$ DOX loaded hydrogel with radiation, $I R+A u+D O X(G)$ AuNPs and DOX co-loaded hydrogel with radiation, $I R+$ $A u(S)$ AuNPs solution with radiation, $I R+D O X(S)$ DOX solution with radiation, $I R+A u+D O X(S)$ AuNPs solution and DOX solution mixture with radiation. ${ }^{\#} p<0.05,{ }^{\# \#} p<0.01$, statistically significant compared with saline treatment

applied on B16 cells and HepG2 cells for the following treatment to get similar cell viabilities on the two cell lines, thus yielding an appropriate absorbance in MTT assay. From the results in Fig. 4a, c, and d, it could be suggested that AuNPs were radiosensitive and that the sensitization ability positively related to AuNPs rather than X-ray doses. Notably, the sensitive effect was less evident in HepG2 cells under 4 or 6 Gy radiation. These could be explained by the different radiosensitivities of different cells and by the different cellular uptake rates of AuNPs on different cells (48.5\% for B16, $28.9 \%$ for HepG2 at 24 h) $(4,29,39)$. Previous studies on cell viability were mostly carried out with X-ray dose constant or changed without a gradation (38). This study, however, gave more details on the relationship of sensitization activity not only with AuNP concentrations but also with the X-ray doses, offering more information in understanding the radiosensitization mechanism. Cell surviving curve was used to describe the relationship between the radiation dose and the proportion of cells that survive $(40,41)$. Radiosensitization activity by AuNPs and the combination effects with DOX were confirmed by comparing the surviving curves in
Fig. 4e, f. Parameters presented in Table III gave the information consistent with that in Fig. 4e, f. Therefore, AuNPs used in this study could efficiently enhance the radiation effect on cells, and the combination treatment by AuNPs and DOX under radiation was effective to inhibit tumor cell growth and decrease the surviving fraction. However, results on HepG2 indicated that the radiosensitivity of AuNPs might show different behaviors on different cell lines; the related details remained to be elucidated.

Our results on cells were in good agreement with that in the following in vivo tests. Moreover, by combination of DOX and AuNPs, fewer AuNPs and DOX were required in this study. About $5 \mathrm{mg} / \mathrm{kg}$ AuNPs were used in this study which was much lower than $1 \mathrm{~g} / \mathrm{kg}$ in Chang's early study (10). A recent research applied AuNPs at a dose of about $4.95 \mathrm{mg} / \mathrm{kg}$ per week, which was close to that in this study (35). The dose of DOX in this in vivo study was about $2.5 \mathrm{mg} / \mathrm{kg}$, which was much lower than the usual dose $10 \sim 30 \mathrm{mg} / \mathrm{kg}$ (27). It has been well-known that the management of caner reflects a balance between efficacy and toxicity. By using less DOX and fewer AuNPs, the possible side 
Lung
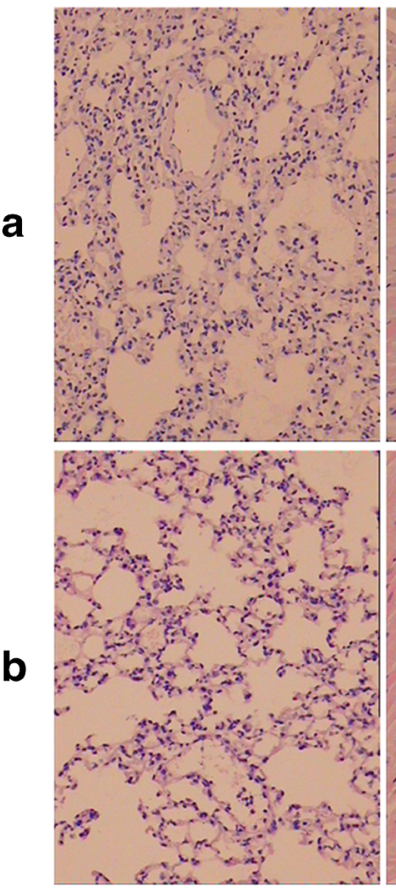

Heart

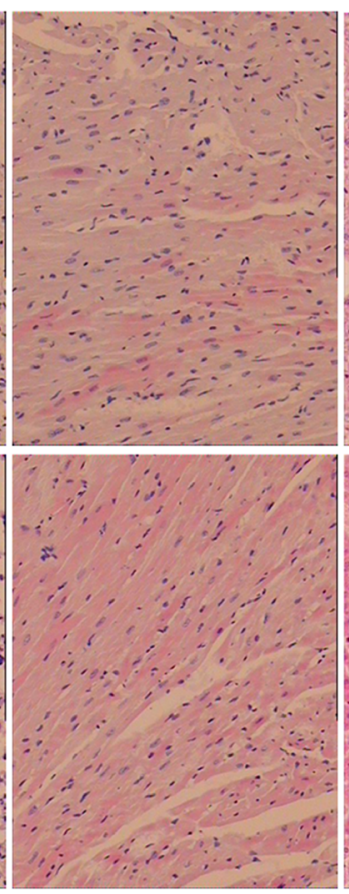

Liver

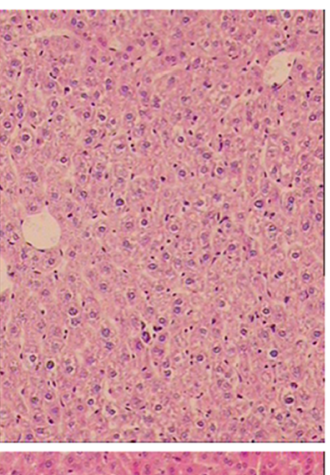

Spleen

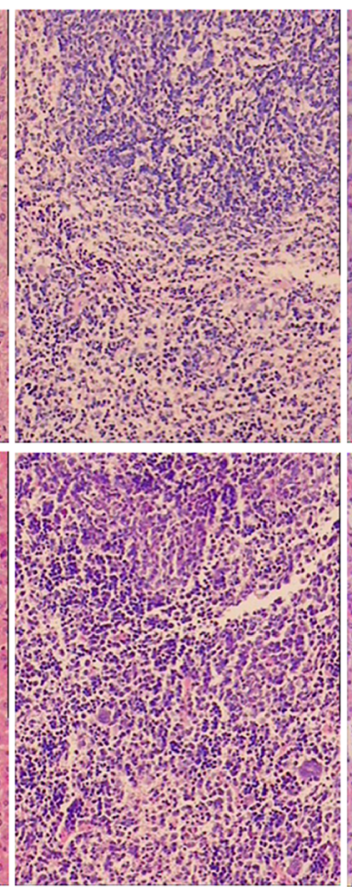

Kidney

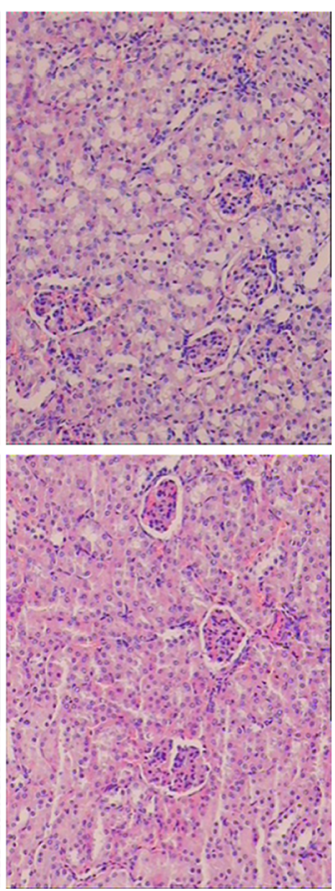

Fig. 7. The hematoxylin-eosin $(H \& E)$ staining of heart, liver, spleen, lung, and kidneys at 20th day post administration: a IR $+A u+D O X(G)$ treatment and $\mathbf{b}$ saline treatment

effects could be alleviated. Results in Fig. 6c revealed the less toxicity of hydrogel than solution, probably due to the slow release of DOX. Therefore, by combination of AuNPs and DOX in a F127-based hydrogel, the tumor could be inhibited efficiently.

Interestingly, AuNPs under radiation condition inhibit the cell growth and tumor cell proliferation, as could be concluded from MTT assays and Ki-67 staining. The proliferation inhibition was increased more after DOX were combined, which proved the combination effects for chemoradiotherapy. The mechanisms by which AuNPs could enhance radiotherapy toxicity are not fully established at present and the findings in this study suggested that the radiosensitization activity and the combination effects with DOX might be caused by the inhibition of tumor cell growth and proliferation (10). Moreover, the AuNP concentrations used in this study were as safe as previously reported $(6,42)$. Primary safety evaluation results showed no visible toxicity of Au-DOX-Gel towards skin, body weight, and organs (heart, liver, spleen, lung, and kidneys).

\section{CONCLUSION}

In conclusion, an enhanced cancer chemoradiotherapy was achieved by the Au-DOX-Gel, in which DOX acted as chemotherapeutics while AuNPs killed tumor cells by enhancing the radiation dose. Sustained release profiles of AuNPs and DOX were testified. The superior radiosensitization effect and anti-tumor effect were verified in vitro and in vivo. Tumor cell growth and proliferation were inhibited by AuNPs combined with DOX under radiation, suggesting that the radiosensitization activity and combination effects might be caused by inhibition of tumor cell growth and proliferation. Overall, the Au-DOX-Gel could be a promising strategy for cancer chemoradiotherapy.

\section{ACKNOWLEDGMENTS}

This work was supported by "The Fundamental Research Funds of Shandong University," 2014QY004.

\section{REFERENCES}

1. Seiwert TY, Salama JK, Vokes EE. The concurrent chemoradiation paradigm-general principles. Nat Clin Pract Oncol. 2007;4(2):86-100.

2. Wang AZ, Tepper JE. Nanotechnology in radiation oncology. J Clin Oncol. 2014;32(26):2879-85.

3. Della Rocca J, Werner ME, Kramer SA, Huxford-Phillips RC, Sukumar R, Cummings ND, et al. Polysilsesquioxane nanoparticles for triggered release of cisplatin and effective cancer chemoradiotherapy. Nanomedicine: NBM. 2015;11(1):31-8.

4. Hall EJ, Giaccia AJ. Radiobiology for the radiologist: Lippincott Williams \& Wilkins; 2006.

5. Wang EC, Min Y, Palm RC, Fiordalisi JJ, Wagner KT, Hyder N, et al. Nanoparticle formulations of histone deacetylase inhibitors for effective chemoradiotherapy in solid tumors. Biomaterials. 2015;51:208-15.

6. Joh DY, Sun L, Stangl M, Al Zaki A, Murty S, Santoiemma $\mathrm{PP}$, et al. Selective targeting of brain tumors with gold nanoparticle-induced radiosensitization. PLOS ONE. 2013;8(4), e62425.

7. Miladi I, Alric C, Dufort S, Mowat P, Dutour A, Mandon C, et al. The in vivo radiosensitizing effect of gold nanoparticles based MRI contrast agents. Small. 2014;10(6):1116-24.

8. Zhang X-D, Wu D, Shen X, Chen J, Sun Y-M, Liu P-X, et al. Size-dependent radiosensitization of PEG-coated gold nanoparticles for cancer radiation therapy. Biomaterials. 2012;33(27):6408-19. 
9. Cooper D, Bekah D, Nadeau J. Gold nanoparticles and their alternatives for radiation therapy enhancement. Front Chem. 2014;2:86

10. Chang MY, Shiau AL, Chen YH, Chang CJ, Chen HHW, Wu CL. Increased apoptotic potential and dose-enhancing effect of gold nanoparticles in combination with single-dose clinical electron beams on tumor-bearing mice. Cancer Sci. 2008;99(7):1479-84.

11. Vilchis-Juárez A, Ferro-Flores G, Santos-Cuevas C, MoralesAvila E, Ocampo-García B, Díaz-Nieto L, et al. Molecular targeting radiotherapy with cyclo-RGDfK(C) peptides conjugated to $177 \mathrm{Lu}$-labeled gold nanoparticles in tumor-bearing mice. J Biomed Nanotechnol. 2014;10:393-404.

12. Park J, Park J, Ju EJ, Park SS, Choi J, Lee JH, et al. Multifunctional hollow gold nanoparticles designed for triple combination therapy and CT imaging. J Control Release. 2015;207:77-85.

13. Stirland DL, Nichols JW, Miura S, Bae YH. Mind the gap: a survey of how cancer drug carriers are susceptible to the gap between research and practice. J Control Release. 2013;172(3):1045-64.

14. Jenkinson MD, Smith TS, Haylock B, Husband D, Shenoy A, Vinjamuri S, et al. Phase II trial of intratumoral BCNU injection and radiotherapy on untreated adult malignant glioma. J NeuroOncol. 2010;99(1):103-13.

15. Kim YH, Gratzinger D, Harrison C, Brody JD, Czerwinski DK, Ai WZ, et al. In situ vaccination against mycosis fungoides by intratumoral injection of a TLR9 agonist combined with radiation: a phase 1/2 study. Blood. 2012;119(2):355-63.

16. Tsao C-T, Kievit FM, Wang K, Erickson AE, Ellenbogen RG, Zhang M. Chitosan-based thermoreversible hydrogel as an in vitro tumor microenvironment for testing breast cancer therapies. Mol Pharm. 2014;11(7):2134-42.

17. Jeong B, Kim SW, Bae YH. Thermosensitive sol-gel reversible hydrogels. Adv Drug Deliv Rev. 2002;54(1):37-51.

18. Sellers DL, Kim TH, Mount CW, Pun SH, Horner PJ. Poly (lactic-co-glycolic) acid microspheres encapsulated in Pluronic F-127 prolong hirudin delivery and improve functional recovery from a demyelination lesion. Biomaterials. 2014;35(31):8895-902.

19. Lin Z, Gao W, Hu H, Ma K, He B, Dai W, et al. Novel thermosensitive hydrogel system with paclitaxel nanocrystals: high drugloading, sustained drug release and extended local retention guaranteeing better efficacy and lower toxicity. J Control Release. 2014;174:161-70.

20. Wang Y, Zeiri O, Neyman A, Stellacci F, Weinstock IA. Nucleation and island growth of alkanethiolate ligand domains on gold nanoparticles. ACS Nano. 2011;6(1):629-40.

21. Zhang L, Liu Y, Yu D, Zhang N. Gadolinium-loaded chitosan nanoparticles as magnetic resonance imaging contrast agents for the diagnosis of tumor. J Biomed Nanotechnol. 2013;9(5):863-9.

22. Lindquist C, Kondziolka D. Advances in radiosurgery: Proceedings of the 1st Congress of the International Stereotactic Radiosurgery Society, Stockholm 1993: Springer Science \& Business Media; 2012

23. Liu J, Yu M, Zhou C, Yang S, Ning X, Zheng J. Passive tumor targeting of renal-clearable luminescent gold nanoparticles: long tumor retention and fast normal tissue clearance. J Am Chem Soc. 2013;135(13):4978-81.

24. Guo H, Zhang D, Li T, Li C, Guo Y, Liu G, et al. In vitro and in vivo study of Gal-OS self-assembled nanoparticles for liver-targeting delivery of doxorubicin. J Pharm Sci. 2014;103(3):987-93.

25. Gobin B, Battaglia S, Lanel R, Chesneau J, Amiaud J, Rédini F, et al. NVP-BEZ235, a dual PI3K/mTOR inhibitor, inhibits osteosarcoma cell proliferation and tumor development in vivo with an improved survival rate. Cancer Lett. 2014;344(2):291-8.

26. Yasmin Z, Khachatryan E, Lee Y-H, Maswadi S, Glickman R, Nash KL. In vitro monitoring of oxidative processes with self- aggregating gold nanoparticles using all-optical photoacoustic spectroscopy. Biosens Bioelectron. 2015;64:676-82.

27. Dong D, Gao W, Liu Y, Qi X-R. Therapeutic potential of targeted multifunctional nanocomplex co-delivery of siRNA and low-dose doxorubicin in breast cancer. Cancer Lett. 2015;359(2):178-86.

28. Liu Y, Feng L, Liu T, Zhang L, Yao Y, Yu D, et al. Multifunctional $\mathrm{pH}$-sensitive polymeric nanoparticles for theranostics evaluated experimentally in cancer. Nanoscale. 2014;6(6):3231-42.

29. Arienti C, Zoli W, Pignatta S, Carloni S, Paganelli G, Ulivi P, et al. Efficacy of different sequences of radio- and chemotherapy in experimental models of human melanoma. J Cell Physiol. 2014;229(10):1548-56.

30. Hong YS. Oxaliplatin, fluorouracil, and leucovorin versus fluorouracil and leucovorin as adjuvant chemotherapy for locally advanced rectal cancer after preoperative chemoradiotherapy (ADORE): an open-label, multicentre, phase 2, randomised controlled trial. Lancet Oncol. 2014;15:1245-53.

31. Mesía R, Henke M, Fortin A, Minn H, Yunes Ancona AC, Cmelak A, et al. Chemoradiotherapy with or without panitumumab in patients with unresected, locally advanced squamous-cell carcinoma of the head and neck (CONCERT-1): a randomised, controlled, open-label phase 2 trial. Lancet Oncol. 2015;16(2):208-20.

32. Akash MSH, Rehman K. Recent progress in biomedical applications of Pluronic (PF127): pharmaceutical perspectives. J Control Release. 2015;209:120-38.

33. Xie Y, Tang J, Lu Z, Sun Z, An L. Effects of poly(propylene oxide)-poly(ethylene oxide)-poly(propylene oxide) triblock copolymer on the gelation of poly(ethylene oxide)-poly(propylene oxide)-poly(ethylene oxide) aqueous solutions. J Macromol Sci. 2013;52(9):1183-97.

34. Akash MSH, Rehman K, Chen S. Pluronic F127-based thermosensitive gels for delivery of therapeutic proteins and peptides. Polym Rev. 2014;54(4):573-97.

35. Zhang $X$, Teodoro JG, Nadeau JL. Intratumoral golddoxorubicin is effective in treating melanoma in mice. Nanomedicine: NBM. 2015;11(6):1365-75.

36. Ruan S, Yuan M, Zhang L, Hu G, Chen J, Cun X, et al. Tumor microenvironment sensitive doxorubicin delivery and release to glioma using angiopep-2 decorated gold nanoparticles. Biomaterials. 2015;37:425-35.

37. Ciombor KK, Yang F, Iii ABB, Su Y, Horton L, Short SP, et al. Phase II trial of bortezomib plus doxorubicin in hepatocellular carcinoma (E6202): a trial of the Eastern Cooperative Oncology Group. Investig New Drugs. 2014;32(5):1017-27.

38. Wang C, Li X, Wang Y, Liu Z, Fu L, Hu L. Enhancement of radiation effect and increase of apoptosis in lung cancer cells by thio-glucose-bound gold nanoparticles at megavoltage radiation energies. J Nanoparticle Res. 2013;15(5):1-12.

39. Choi Y, Kim JW, Cha H, Han KH, Seong J. Overall response of both intrahepatic tumor and portal vein tumor thrombosis is a good prognostic factor for hepatocellular carcinoma patients receiving concurrent chemoradiotherapy. J Radiat Res. 2014;55(1):113-20.

40. Chang DS, Lasley FD, Das IJ, Mendonca MS, Dynlacht JR. Fractionated radiation survival models. Basic Radiotherapy Physics and Biology: Springer; 2014. p. 221-33.

41. Yasui H, Takeuchi R, Nagane M, Meike S, Nakamura Y, Yamamori $\mathrm{T}$, et al. Radiosensitization of tumor cells through endoplasmic reticulum stress induced by PEGylated nanogel containing gold nanoparticles. Cancer Lett. 2014;347(1):151-8.

42. Simpson CA, Salleng KJ, Cliffel DE, Feldheim DL. In vivo toxicity, biodistribution, and clearance of glutathione-coated gold nanoparticles. Nanomedicine: NBM. 2013;9(2):257-63. 